\title{
Exploring Digital Image Frames for Lifestyle Intervention to Improve Well-being of Older Adults
}

\author{
Andreas Seiderer, Stephan Hammer, \\ Elisabeth André \\ Augsburg University \\ \{seiderer,hammer,andre\}@hcm-lab.de
}

\author{
Marcus Mayr, Thomas Rist \\ Augsburg University of Applied Sciences \\ \{thomas.rist,marcus.mayr\}@hs- \\ augsburg.de
}

\begin{abstract}
This contribution addresses the development of technology for senior users with the aim to improve their general wellbeing. We present a prototype system named CARE that is used for in-situ testing in a senior's home and combines functionality of a digital image frame with an active recommender mode. The purpose of the recommender is to provide the user with context-specific recommendations. Recommendations are chosen on the basis of sensor data and a well-being model to carefully decide on at which point in time what kind of activity will be most suitable to suggest.
\end{abstract}

\section{Categories and Subject Descriptors}

H.5 [Information Interfaces and Presentation]: Miscellaneous; J.3 [Life and Medical Sciences]: Health

\section{INTRODUCTION}

Increases in longevity and improvements in health care services together with declining birth rates contribute to the irresistible phenomenon of an aging society in almost all western countries. While higher life expectancy is a positive achievement, it comes along with societal challenges, such as social isolation, loss of independency, inactivity and missing initiative of elderly people. Lifestyle choices are among the most important factors that determine a person's well-being and quality of life. Studies have shown that regular physical exercise can help mitigate many age-related diseases. Furthermore, creative activities, such as painting, have a positive influence on well-being. Studies conducted in a senior citizen's apartment building have shown that interventions that encourage the elderly to participate in activities, such as taking over responsibility for household chores, have been effective means to prevent social isolation and loneliness.

The objective of CARE (a sentient Context-Aware Recommender system for the Elderly) is to develop a sensitive and personalized assistance system to support single-living people in their domestic environment. Assistance is given in

(c) Author | ACM 2015. This is the author's version of the work. It is posted here for your personal use. Not for redistribution. The definitive Version of Record can be found at http://dx.doi.org/10.1145/2750511.2750514 the form of situationally appropriate and unobtrusive recommendations to encourage physical, mental and social activities, all aimed at increasing individual quality of life and vitality. The current paper reports work on a first CARE prototype system. After discussing related work, we provide an overview of the overall CARE concept and its implementation in terms of soft- and hardware. A first trial run of CARE has been conducted over a period of two weeks.

\section{RELATED WORK}

There are a growing number of projects that aim to promote a healthier lifestyle and to increase quality of life and overall well-being. Many systems rely on self-monitoring as a means to evoke a behavior change in people. Typically, health-related data are presented to the user in terms of diagrams to make the users reflect on them. Thereby, data are either manually collected by the users themselves or automatically acquired from body-worn sensors, or sensors embedded in the environment or in the users' mobile phone.

Since many users have difficulties to draw relevant conclusions from diagrams, attempts have been made to convey health-related data in a form that is easier to grasp. The BeWell system [9] represents different well-being dimensions, such as the users' physical activities or their sleeping patterns, by different characters in an aquatic ecosystem. The UbiFit system [3] employs the metaphor of a virtual garden that reflects the user's physical fitness. The Health Mashups system [2] makes use of natural language to show users how their well-being is connected to their lifestyle. Information on these correlations is derived from personal user and context data collected with sensors integrated into the users' mobile phone. For example, users may be told that they tend to feel better if they have more sleep. The Motivate system [11] maps health-related data about user and context directly onto situated recommendations based on the consideration that advice is more likely to be followed if it is presented at the right location and the right point in time. López-Nores et al. [12] explored possibilities to incorporate health-related recommendations into semantically matching TV programs.

The work presented here also bears resemblance to several prior projects in home-based monitoring, such as MIT's PlaceLab [5] or the Georgia Tech Aware Home [7]. However, while the objective of these projects is to explore a variety of sensing technologies for smart homes, we focus 
on the question of how to enhance well-being by providing context-sensitive life style recommendations.

Unlike most lifestyle and health monitoring systems, CARE specifically focuses on seniors from the $65+$ age group as target users. This has various implications with regards to sensor data acquisition, data interpretation, and the kind of recommendations that CARE delivers to its users.

\section{WELL-BEING FACTORS}

A number of attempts have been made to define what factors constitute a person's subjective feeling of well-being, and to identify suitable indicators for measuring subjective well-being, e.g. for the purpose of comparisons. As it is often the case with high-level socio-physiological constructs, there is little consensus regarding the definition of this construct and its differentiation from related constructs, such as "happiness", or "Quality of Life" which is in focus of various studies in the medical domain. The great diversity in the definition of constructs and models is mirrored by the broad range of different survey questionnaires and online tools for measuring a person's degree of well-being, happiness, or health-related-quality of life. For example, a large collection of such surveys is accessible on the website of the UK's Office for National Statistics (www.ons.gov.uk), and the website www.energystrategy.eu lists a number of surveys for self-testing. A profound comparison of models is beyond the scope of our work. Rather, we take a pragmatic approach and -as a starting point for our work towards a CARE prototype- we oriented ourselves on a model that has been put forward by the new economic foundation. On their web-site "National Accounts Well-Being" (www.nationalaccountsofwellbeing.org) they report on an initiative which aimed to measure subjective well-being of citizens living in different European countries. Their underlying conceptualization of well-being is based on personal well-being and social well-being as headline indicators, each of which is broken further down into a number of sub- and sub-sub-categories. At the lowest level of this hierarchy, categories are linked to concrete items of a questionnaire that can serve as a measuring tool in well-being studies.

For CARE we also rely on a hierarchical model of wellbeing factors. For practical reasons, however, we use a simplified model, it includes only categories which are linked to recommendations that a system like CARE is able to deal with. For instance, CARE can make suggestions about how to improve physical fitness, but it would be difficult to provide recommendations regarding a senior's income which is considered a dominating factor for economical well-being.

Our current model comprises the following headline categories: physical, mental, social, emotional, and environmental well-being. As shown in Fig. 1, these categories are further broken down into subcategories. In the context of CARE, the purpose of a well-being model is to associate with its subcategories a set of dedicated recommendations that may be presented to a senior, in the hope that recommendations are followed and that recommended activities eventually contribute positively to well-being (cf. Sec. 4).

It is important to note that our objective was not to come up with a new model of well-being factors, but to adjust existing models to the specific requirements of CARE and check its plausibility for our target users. To this end, we conducted structured interviews among a peer-group of 21 seniors (13 female, 8 male, in the age range between 67 and
83), all living either alone or together with a partner in an own household. The interviews started with a number of demographic questions (including age, gender, education, marital status, accommodation, and living arrangements) and questions on usage of electronic media (TV, radio, computer, internet, and digital picture frames) at home. An important outcome for the development of CARE was that all participating seniors had an idea of what a digital picture frame is, though only two of them had one at home.

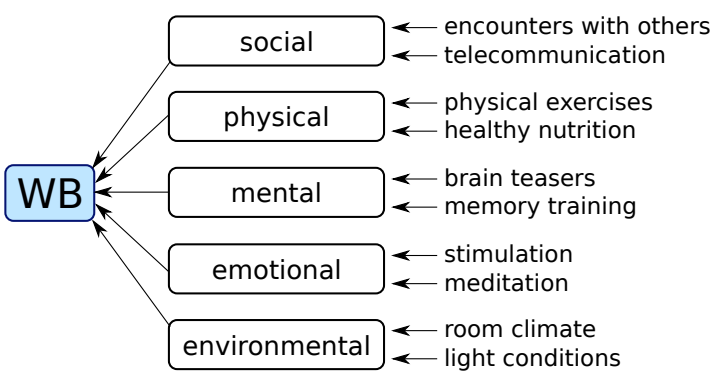

Figure 1: CARE's model of well-being factors

The interviews continued with specific questions related to the subcategories of CARE's well-being model. The objective of this part was to learn about:

- the activities that seniors already perform regularly, and that could relate to the model's subcategories

- what importance the seniors attach to those activities

- whether the seniors have set specific targets to engage more strongly / often in those activities

- whether the seniors see specific obstacles that may hinder stronger engagement

For illustration, let's focus on the subcategory physical well-being. This subcategory depends, among other factors, on a person's physical fitness (or physical vitality), the general health condition, and feeling of vigilance.

Physical fitness may be measured by indicators, such as endurance, strength, coordination, and suppleness. To assess a person's physical fitness, we asked people which kind of physical activity they perform and how frequently they do so. We also asked whether it is important to them to be physically active, whether they strive for increasing their physical fitness to improve their overall well-being, and if they face specific obstacles (e.g. indispositions) which hinder them to engage more in physical activities. Our participants said that physical fitness was important to them $(\mathrm{M}=3.7, \mathrm{SD}=1.342)$. Most seniors said that they wished to improve their physical well-being by more physical activities and by paying more attention to healthy nutrition. It turned out that seniors who engaged frequently in physical activity (e.g. riding a bicycle, climbing stairs or going out for walks) felt themselves physically more fit than others. As barriers the seniors mentioned physical impairments at first place, but also a lack of time due to other obligations (e.g. caring for relatives or pets). These results are in line with a study performed by Fan et al. [4].

Our participants gave high importance to feeling vigilant $(\mathrm{M}=4.55, \mathrm{SD}=0.51)$. To assess people's feeling of vigilance we asked the seniors how often they feel tired during the day, 
and how frequent they suffer from insomnia. We also asked whether they take any counter measures (such as drinking coffee, go out to breath fresh air, take a shower, or having a nap). Most people (15 out of 21) indicated that they take a nap. Only a few people (6 out of 21 ) said that they go out to get some fresh air. As reasons for being tired, the participants indicated urge to urinate, noisy neighbors, medicaments, joint pain, or the use of a breathing device.

We noticed a strong correlation between general health condition and physical fitness (Pearson Product-Moment Correlation: $\mathrm{r}=0.94 ; \mathrm{p}=0.001)$, a medium negative correlation between general health condition and the frequency of feeling unconcentrated $(\mathrm{r}=0.58 ; \mathrm{p}=0.007)$.

Interestingly, the survey also revealed that "healthy food" matters when speaking about well-being. Therefore, our model considers "healthy nutrition" as a contribution factor to physical well-being.

For mental well-being we assume mental fitness as a determining factor with measurable indicators, such as capacity for remembering, creativity, logical thinking and inference capability. We did not ask subjects for a self-assessment of mental fitness, but asked them how frequently they forget names, phone numbers, and dates, or need to search for objects because they forgot where they have placed them. Most seniors said that they engage in specific activities to improve mental fitness (such as solving quizzes or crossword puzzles, or memorize numbers). Factors negatively affecting memory and concentration mentioned by the seniors included: headaches, uneasy sleep, stress, medicaments and age.

With regard to social well-being, we asked questions concerning the frequency of face-to-face encounters with relatives, friends, and neighbors, how often they receive and make phone calls, and how often they go out for attending public events. Our participants gave high importance to being in touch with family members and friends $(\mathrm{M}=4.8$, $\mathrm{SD}=0.696$ ). Half of the participants (10 out of 21 ) were in daily contact with family members, friends or neighbors. As barriers to meeting people, the seniors mentioned missing means of transport, tiredness and weariness.

Regarding the subcategory environmental well-being we asked how satisfied they were with their housing condition and their living environment (e.g., the opportunities for shopping, medical treatment, recreation and leisure activities, and contentedness to public transportation). Here, most participants gave a neutral rating suggesting that they were not aware of the offers their quarter provides.

For the subcategory emotional well-being we only included a few general questions, such as whether they perform relaxation or meditation exercises. We found a medium negative correlation between general health condition and the frequency of feeling stressed $(\mathrm{r}=0.56 ; \mathrm{p}=0.01)$.

In principle, responses to the indicators of certain wellbeing categories could be aggregated into a numeric score for measuring a person's well-being. Such scores could be used for assessing the success of a system like CARE. However, this was beyond the objective of our current work.

\section{WELL-BEING RECOMMENDATIONS}

The underlying rationale of the CARE approach is that general well-being of elderly users can be positively influenced by means of certain activities which are recommended or stimulated through CARE during the day. However, to achieve this objective a number of prerequisites have to be met:

- P1: recommended activities must be related to one or more factors determining a person's well-being

- P2: recommendations must be of relevance for the person at the time when they are given

- P3: the person must be able to follow them

- P4: the person must be motivated to do so.

Regarding requirement $\mathrm{P} 1$, we started from the well-being model introduced above and determined for each sub-category of the model a set of activities that may be recommended.

Activities to improve physical well-being: According to our well-being model, physical well-being is the "netimpact" of physical fitness, general health condition, vigilance / alertness, and nutrition.

Physical fitness in terms of endurance, muscle strength, coordination and flexibility can be improved by means of physical activity. In fact, there is a myriad of books, video, and online resources recommending dedicated exercises to improve physical fitness, and the number of those being targeted towards elderly people is rapidly growing. For the purpose of CARE we distinguish between the general term physical activity and exercises. All activities that require body movement and increased energy expenditure are subsumed by the term physical activity. This includes everyday activities, such as climbing stairs, walk on foot for shopping, do gardening etc., as well as leisure-oriented activities, such as going out for a walk in the park, or have a swim in the pool. In contrast, an exercise is as structured, typically repetitive body movement which serves a specific purpose, such as strengthening muscle power (strengthening exercises), widening the range of motion around joints (flexibility exercises), improving cardio-respiratory endurance (aerobic exercises), or to improve coordination and balance (coordination exercises), e.g. for fall prevention.

Fig. 2 and 3 show some sample recommendations. To serve their purpose, the elements of a recommendation are associated with certain communicative functions. For example, the recommendations in the left part of Fig. 2 and 3 instruct a finger coordination and a strengthening exercise. The instructions about what to do are given by the text, whereas the images provide additional information on how to perform the exercise. In contrast, the recommendation in the right part of Fig. 2 stimulates a walk outside in the park.

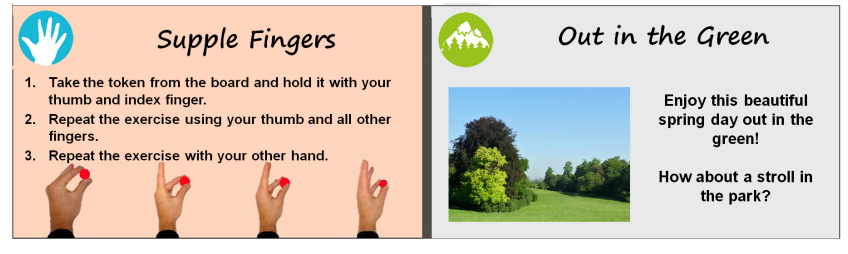

Figure 2: Recommendations to stimulate physical activity

The recommendation shown in the right part of Fig. 3 is associated with the sub-category healthy nutrition. It reminds a person to drink enough and may be displayed especially after physical exercises. 


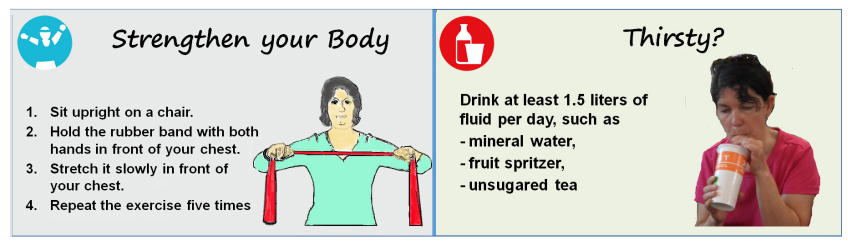

Figure 3: Recommendations to improve physical well-being

To facilitate the recognition of the type of a recommendation we use a common headline text and a logo for recommendations of a certain type, such as finger or strenghtening exercises, outdoor activities, or diet and drinking recommendations.

Activities to improve mental well-being: It has been shown that regular physical activity has protective benefits for the brain. In a cohort study with 1,740 adults older than 65 Larson et al. [10] found that the incidence of dementia in individuals who walked three or more times per week was $35 \%$ lower than those in individuals who walked less than three days per week. This suggests that recommendations for physical activity improve physical fitness and at the same time can contribute positively to mental fitness.

During the last decade a plethora of mental fitness software entered the consumer market, often especially targeted at senior users who are afraid of cognitive decline. The assumption and promise of these products is that mental training strengthens cognitive abilities, such as memory, attention, language processing, visual-spatial skills, logical reasoning, planning etc. While repeated / regular engagement in mental fitness exercises often yield observable improvements in task performances, it is less clear to what degree positive training effects transfer to everyday activities, and improve mental fitness in general (cf. [15]).

CARE's set of mental exercises is inspired by the so-called Mini Mental State Examination (MMSE) which was developed as a tool for care givers to assess an older adult's mental status [13]. It comprises 11 tasks to assess five areas of cognitive function: orientation, registration, attention and calculation, recall, and language. For CARE we formulated quizzes that relate to these cognitive functions. To make the quizzes more relevant to the user, reference to the users' context is made where possible. For example, rather than having the user memorize an arbitrary sequence of numbers, CARE would ask the senior to recall a certain phone number (see Fig. 4, left) of a family member, or the date of a past event. A typical orientation task is shown in the right part of Fig. 4. The quiz shows a prominent landmark in the senior's neighborhood and asks for a way direction.

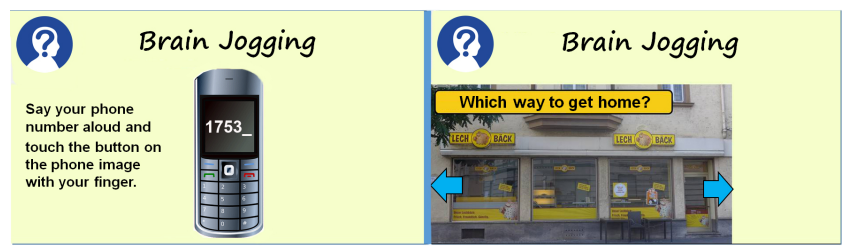

Figure 4: Quizzes to train cognitive skills

Activities to improve emotional well-being: Emotional well-being refers to the emotional quality of an indi- vidual's everyday experience, i.e., the frequency and intensity of unpleasant and pleasant feelings (cf. [6]). Hence, activities that stimulate positive affect or reduce negative affect are candidates for recommendations [14]. The recommendation shown in the left part of Fig. 5 is an explicit request to take a smile. Further recommendations associated with emotional well-being are jokes or funny riddles.

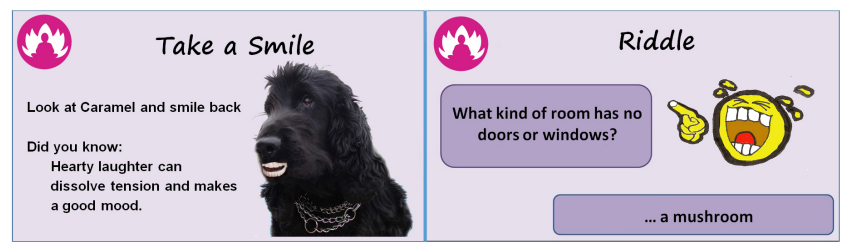

Figure 5: Activities to improve emotional well-being

Activities to improve social well-being: Potential candidates for this well-being factor are activities that initiate, maintain, widen, or intensify interpersonal relationships. As many seniors living apart from their children and grand children wish to maintain close contacts to them but are often reluctant to take initiative without having a specific reason. Therefore, CARE's repertoire of recommendations includes reminders to family events which are opportunities to make a phone call. A typical example is a "birthday reminder" as shown in the left part of Fig. 6 .

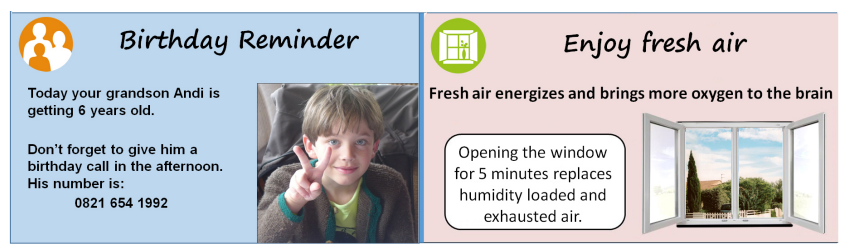

Figure 6: Activities to improve social (left) and environmental (right) well-being

Activities to improve environmental well-being: The recommendation shown in the right part of Fig. 6 may be triggered if indoor air quality falls below a certain threshold. It is a recommendation to air the room as suggested by the image of an open window. There is no textual request to air the room. Rather, the headline and the text just provide motivating arguments for letting in fresh air.

\section{OVERVIEW OF THE CARE SYSTEM}

The CARE system capitalizes on the concept of a digital picture frame as such devices are often given to senior family members. However, CARE makes a substantial extension to the classical concept. It interleaves the display of photos with physical exercises, brain-twisters and situation-specific life style recommendations, in other words, it combines two modes: Picture frame and Recommender mode (cf. Fig. 7).

The Picture frame mode is activated when the user is further away from the display. In this mode, CARE appears to the user like an ordinary picture frame which displays photos that are either taken from local repository, or which are remotely uploaded by family members via an internet connection. Users implicitly get information through a quick glance. However, they neither control the display of pictures nor do they interact with the system. 


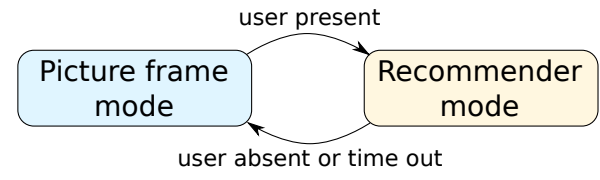

Figure 7: CARE system states

The Recommender mode is triggered by user presence detected in front of the display. In this mode, users receive context-specific recommendations. Recommendations are chosen on the basis of data acquired by sensors embedded in the user's environment and possibly body-worn sensors as well as a user model, a discourse model, and a model of well-being factors to carefully decide on at which point in time what kind of activity will be most suitable to suggest in order to increase the user's well-being.

In order not to overstrain users, interaction with the display is deliberately kept simple. Users are able to interact with the display, for example, by selecting buttons to answer quizzes. Furthermore, they can provide feedback on presented recommendations and indicate how they feel by selecting specific icons on the screen.

Fig. 8 gives an impression of the CARE prototype. Its hardware consists of a tablet computer with a touch screen (a low-priced HP Omni 10 5600eg running Windows 8.1) that has been integrated into a magnetic pin board. For the purpose of testing, the board is mounted on two rails so that the height of the display screen can be adjusted for its users. The tablet itself has the following integrated sensors: accelerometer, gyroscope, microphone, light sensor, compass, camera and inclinometer. The pin board is equipped with further sensors. An infrared (IR) distance sensor is used to detect the presence of a person in front of the device within a detection range of 20 to $150 \mathrm{~cm}$. A magnetic field sensor and a sensor for measuring air quality are mounted at the backside of the device. The magnetic field sensor (a 3 -axis magnetometer) detects changes in the magnetic field that occur when magnets are removed from or added to the pin board. For measuring indoor air quality we rely on an iAQ-engine sensor which is embedded in a USB stick and connected to CARE.

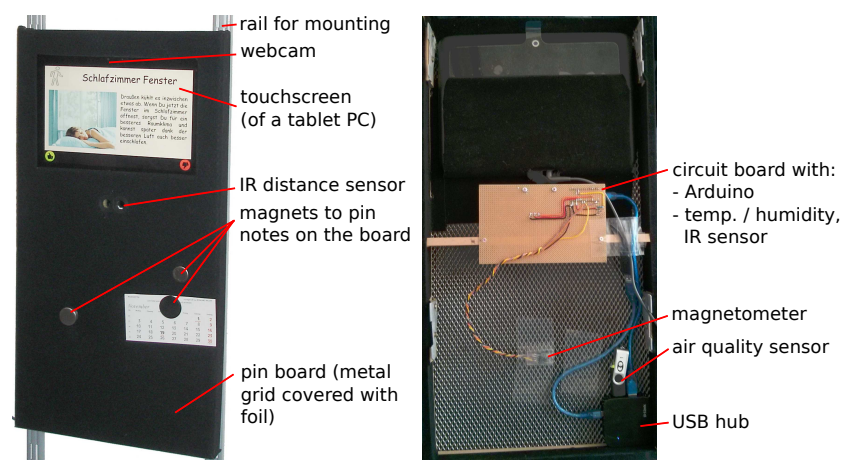

Figure 8: Front- and back-side view

Fig. 9 shows the core software components and knowledge sources of the CARE system. The sensor acquisition component interfaces with all attached sensors collects multisensory data with sensor-specific sampling rates. To reduce the volume of raw data, sensor readings with sampling rates higher than $2 \mathrm{~Hz}$ are aggregated (using aggregation functions, such as max, min, mean, median, or sum). Aggregation is also applied for privacy reasons. For example, from recorded audio data CARE only considers intensityrelated audio features, such as averaged acoustic amplitudes, for further processing. The camera the tablet is equipped with could be used for activity recognition without saving the raw data. However, this feature has not yet been integrated in the current CARE prototype due to privacy concerns expressed by the users. We use dedicated classifiers and mechanisms for fusing sensor data to obtain relevant context information. For example, CARE must be aware of whether a person is in the room where the system is installed and whether a person is getting close to the CARE installation. To this end, following sensor readings are exploited: IR distance, noise level, air quality, temperature, humidity, brightness and magnetic field. The presence of a person in the room may be detected by a higher level of background noise, and after a certain time also by a higher concentration of carbon dioxide and volatile organic compounds which is recorded by the air quality sensor (for details see Sec. 6.2). In the evening hours and during the night, an abrupt change of illumination provides further evidence of someone entering or leaving the room. From the readings of the IR distance and the light sensor it can be inferred that a person is in the vicinity of the CARE installation, and thus may take notice of what is displayed on the screen. Also, physical interaction with the magnetic pin board can be detected by the magnetometer.

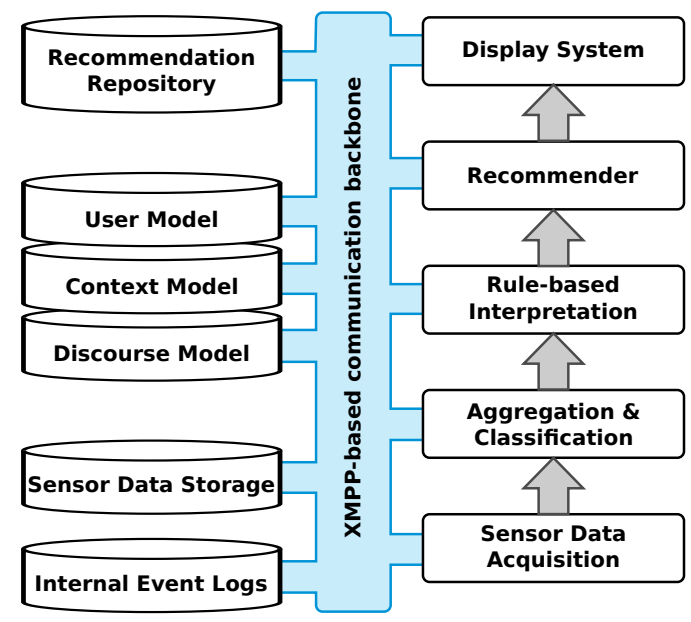

Figure 9: Core software architecture.

A central task of CARE is to decide on when to switch from picture frame mode to recommender mode. For the sake of simplicity, the first CARE prototype switches to recommender mode every time a user is detected in front of the display for a minimum of two seconds.

When switching to recommender mode, the recommender module is called to select a recommendation for display. The selection approach is a two-step procedure. First a rulebased, contextual pre-filtering approach [1] is implemented to filter out inadequate recommendations. It considers the current time of day, the local sunrise and sunset times and the current weather conditions. For example, if it is dark outside, all outdoor activities, such as "take a walk", are filtered out. After pre-filtering, the recommender system 
chooses a single recommendation while considering the prior display history. Furthermore, the recommender module is able to immediately react on particular situations by triggering a corresponding rule. For example, if the trigger event refers to bad air quality in the room, the module selects a recommendation of category "environmental well-being". At specific times during the day, users are encouraged to provide information on their current mood by pressing specific buttons. In case of a bad user mood, recommendations of the category "emotional well-being" have an increased relevance and will get selected with a high probability.

Selected recommendations are forwarded to the display system. A recommendation is displayed to the user for a predefined time period. The duration depends on the type of recommendation and its presentation format. For example, recommendations for physical exercises are typically presented for 60 seconds to give the user enough time to read the instructions and to perform the exercise. After showing a recommendation to the user, the display switches back to the implicit mode. Currently, the system's possibilities to find out whether a recommendation has been followed are still limited. The pin board includes a set of accessories that are attached with magnetic pins. Using the magnetometer, interactions with the magnetic pins can be detected. For example, if a stretch band exercise is recommended and the magnetic field sensor detects a change in the magnetic field, the system takes this as evidence that the user took the band from the board. If the user answers to a quiz or rates a recommendation item, this information is stored in the user model. Currently, this information is not utilized in the recommendation process. However, it is used to analyse the user's preferences and the recommender system's performance and persuasiveness offline and to develop a more sophisticated recommender system.

For inter-module communication we rely on the XMPP protocol with messages in JSON format. This allows platform independent SSL encrypted communication, and facilitates integration of further modules, even if they are executed on different nodes of a computer network. All modules produce various notifications which are stored in an event log database. These log data facilitate debugging but can be exploited for evaluation purposes as well.

\section{IN-SITU INSTALLATION}

The ultimate objective of CARE is to contribute positively to a senior person's well-being through lifestyle interventions provided during the day. Demonstrating such an effect is very challenging. Based on the observation that showing a behavior change is often infeasible at an early stage of system development, Klasnja et al. [8] argue that focusing on this kind of evaluation is too limited. As a first step of system evaluation, they therefore promote studies that contribute to a deeper understanding of how a health care system is being used. Following their argumentation, our first in-situ test focused on the feasiblity of the CARE approach. In particular, we were interested in finding out whether seniors noticed recommendations provided by CARE and identifying typical patterns of use.

To this end, we did a pilot installation of our first CARE prototype and logged system usage over a period of 14 days. The in-situ evaluation took place in the apartment of an elderly couple, a 76 years old female (named " $F$ " in the sequel), and a 75 years old male (named "M").
First, a suitable location for mounting CARE had to be found. The couple inhabits an apartment with a living room, a bed room, a bath room, a kitchen, and a small hallway. We explained to them that the system should be placed best at a location that meets the following requirements:

- CARE should be mounted in a room in which they like to dwell (e.g., the living room or the kitchen).

- The display should be mounted at eye level and be easily accessible (i.e., access to CARE shouldn't be blocked by furniture or by an opened door or window).

Eventually it was agreed on mounting CARE at a kitchen wall close to the passage to the living room. The CARE staff member who launched the system advised the couple that they should not try to switch off the running system or unplug the cable from the power outlet. Instructions on how to use CARE, or comments on CARE were not given. During the test period, the developers checked the system's functions twice, produced backups of recorded data and restarted the system. Except for these two interventions, the system was running continuously. In addition, we gave each senior a wristband equipped with an AX3 data logger, and asked them to wear the bands as often as possible since this would give us valuable data for our study. At this point we were only interested in finding out whether deployment of body worn sensors would be a realistic option for a further CARE prototype in terms of user acceptance.

\subsection{Encounters with CARE - Usage Scenarios}

Fig. 10 gives an impression on how users experienced and interacted with CARE while it was installed at their apartment. When a person is noticed in the close vicinity of CARE, the system switches from passive to active mode (cf. 10a). Such a state change is indicated to the user by swapping the displayed content - the ambient background image gets replaced by the display of a recommendation which has been selected by the recommender engine. The recommendation remains on the screen until the user provides explicit feedback (cf. 10b), or until a timeout is reached, or in case the user is no longer in the range of CARE's sensory system for detecting the presence of a person. This is taken as that the user went away, or is at least no longer close enough to read the displayed recommendation. Note that $\mathrm{F}$ was wearing the AX3 wristband (cf. Fig. 10b).
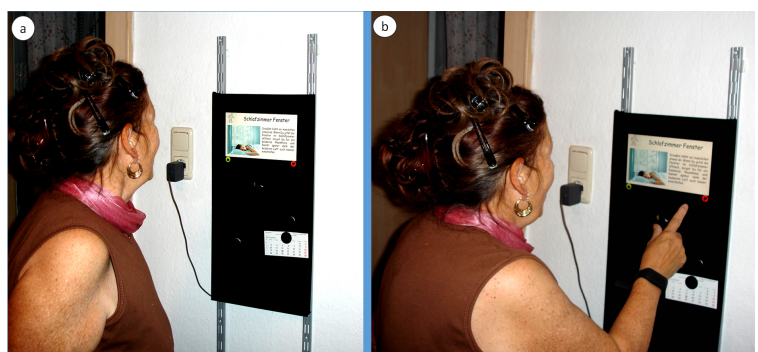

Figure 10: Senior $F$ reading a recommendation

First feedback by the two users revealed that CARE integrated well into their daily routine. In particular, the female user found the recommendations for physical exercises very useful, which helped her stay active. However, we also 
observed some timing issues with the display of recommendations. For example, the female user said that she would like to conduct longer exercises on a regular basis, about 20 to 30 minutes, twice a day. Overall, the duration of the interaction was considered too short. Furthermore, the users wanted more control over the interface. In particular, better mechanisms are required to adapt the timing of the presentations to the users' needs and preferences.

\subsection{Analysis of data logs}

During operation, the CARE system performs intensive logging of data which originate from connected sensors, and CARE system modules. All entries of CARE's internal event log database are time-stamped and listed in temporal order. In particular, the following information has been logged:

- Recommendations that have been presented,

- Assessment of a recommendation by the user,

- Self-assessment of the user's emotional well-being,

- Sensor readings (e.g., temperature, humidity, IR distance and 3-axis magnetometer),

Log-file data are used to identify and characterize usersystem encounters during the test period. We wanted to know whether the users take notice of CARE when in the room where the system was installed. To this end, we need to detect the user's presence in the room (UPRoom) and in front of CARE's display screen (UPScreen).

To determine an UPScreen event we rely on the IR sensor signal amplitudes. If we observe a high amplitude for a period of at least two seconds, we assume that a user dwells in front of the screen and takes notice of the displayed content. Short IR sensor signal amplitudes are typically caused by a user passing by (probably without taking notice of the screen) or by sensor noise. During the test period, about $29 \%$ of observed periods of high IR signal amplitudes were classified as UPScreen events. In addition, the top row of Fig. 11 shows the mean daily distribution of high IR sensor amplitudes and the portion of periods classified as UPScreen events. The diagram also shows that encounters with CARE during a day typically occurred between $8 \mathrm{am}$ and $11 \mathrm{pm}$.

For UPRoom events, the implication holds: "If UPScreen then UPRoom". Short periods with high IR signal amplitudes may be interpreted as a person passing by which means that the person must be present in the room. However, additional sensors are required due to the limited detection range of the IR sensor. In our current system setup we exploit lightness, acoustic noise level, and air quality as additional sources for UPRoom detection.

The lightness sensor can be used to infer about user presence in the room during time periods where artificial light sources are used. Our test trial took place during November in Germany and CARE was installed in a kitchen with two windows through which daylight fell in from about 8am to $4 \mathrm{pm}$. This time period is excluded in the further analysis. For the remaining period (from $4 \mathrm{pm}$ to $8 \mathrm{am}$ of the next day) we extract light switch-on / switch-off events from readings of the light sensor. A switch-on event gives strong evidence for a person entering a room, whereas a switch-off event indicates a person is leaving the room. However, there are cases where a person leaves the room without switching off the light as well as cases where a person enters an already illuminated room. The second row from top of Fig. 11 shows the mean distribution of UPRoom events detected from switchon-light events during a day.

The noise level measured in a room is a further source of information for detecting UPRoom events. Acoustic disseminations may stem from human activity, such as washing and putting away dishes, using kitchen equipment, talking to another person or a pet. For UPRoom detection from noise, peaks in the variance of the noise level above a specific threshold were counted. However, a raw noise level signal alone wouldn't be sufficiently reliable for UPRoom detection for a number of reasons. For example, a fridge located in the kitchen periodically produces noise during the whole day. Fortunately, stable noise sources can be filtered out by using the signal's variance. More difficult are irregular noise sources, such as incoming outdoor noise in case a window has been left open, or when the tv set or radio is turned on in the neighboring living room.

A fourth resource for UPRoom detection is CARE's builtin air quality sensor. It measures $\mathrm{CO}_{2}$ concentration as well as the amount of certain volatile organic compounds (VOCs) in the air, such as cooking odors. As humans exhale $\mathrm{CO}_{2}$ one can expect that a person's presence in a room with closed windows and doors will be mirrored by an increase of the $\mathrm{CO}_{2}$ concentration in that room. In addition, activities, such as cooking significantly increase the VOCs level. An increase of $\mathrm{CO}_{2}$ and VOCs concentration means a decrease of air quality. Thus a drop in air quality may be used for UPRoom detection. The bottom row of Fig. 11 shows the mean daily distribution of UPRoom events detected from air quality. Note that according to the diagram air quality dropped around $4 \mathrm{pm}$. This is consistent with the users' daily routine, i.e., they usually go out for having lunch but prepare a small warm meal in the late afternoon.

An alignment of UPRoom events as detected from readings of all four sensors is shown in Fig. 11. As expected, there are strong positive correlations between the distribution of UPRoom events derived from the IR signal and UPRoom events detected from lightness $(\mathrm{r}=0.94, \mathrm{p}<0.0005)$, noise level $(\mathrm{r}=0.83, \mathrm{p}<0.0005)$ and air quality $(\mathrm{r}=0.82, \mathrm{p}<0.0005)$. In sum, aligned sensor input yields a reliable measure for UPRoom detection.

\section{CONCLUSION}

In this paper, we presented a model of well-being that formed the basis of a life style intervention system for elderly people. A first prototype that combines the functions of a digital image frame with an active recommender mode has been installed in-situ in a seniors' home. The ultimate goal is to contribute positively to a senior person's well-being. As a first step, we investigated how sensor data can be exploited for the identification of characteristic usage patterns. The collected log file data suggest that CARE integrated well into the users' daily routine. Since seniors had to pass it several times a day during their household chores, information could be displayed at an opportune time and place.

Future work will address the involvement of further sensor input. For example, the AX3 sensor can be used to give the user feedback on how well she performs certain physical exercises. With regard to system evaluation, we will concentrate on the question of how to measure the effect of CARE usage on senior people's well-being. 


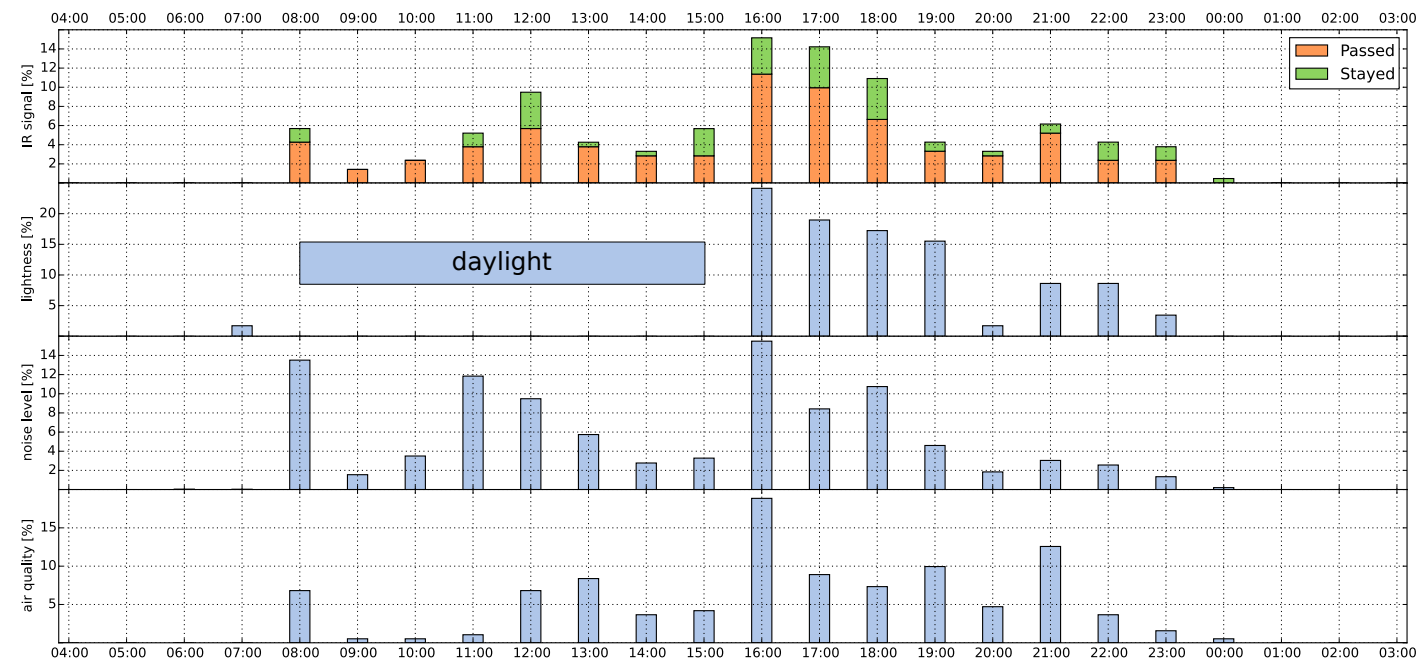

Figure 11: Mean daily user presence distribution using different sensor signals

\section{ACKNOWLEDGMENTS}

This work is supported by the German Federal Ministry of Education and Research (BMBF) under grant number 01XZ13022 and carried out jointly with a sister project at the ICCS of the National Technical University of Athens.

\section{REFERENCES}

[1] G. Adomavicius and A. Tuzhilin. Context-aware recommender systems. In F. Ricci, L. Rokach, B. Shapira, and P. B. Kantor, editors, Recommender Systems Handbook, pages 217-253. Springer US, 2011.

[2] F. Bentley, K. Tollmar, P. Stephenson, L. Levy, B. Jones, S. Robertson, E. Price, R. Catrambone, and J. Wilson. Health mashups: Presenting statistical patterns between wellbeing data and context in natural language to promote behavior change. $A C M$ Trans. Comput.-Hum. Interact., 20(5):1-27, 2013.

[3] S. Consolvo, D. W. McDonald, T. Toscos, M. Y. Chen, J. Froehlich, B. Harrison, P. Klasnja, A. LaMarca, L. LeGrand, R. Libby, I. Smith, and J. A. Landay. Activity sensing in the wild: A field trial of ubifit garden. In Proc. of the SIGCHI Conference on Human Factors in Comput. Systems, pages 1797-1806, New York, NY, USA, 2008. ACM.

[4] C. Fan, J. Forlizzi, and A. Dey. Considerations for technology that support physical activity by older adults. In Proc. of the 14th International ACM SIGACCESS Conf. on Computers and Accessibility, pages 33-40, New York, NY, USA, 2012. ACM.

[5] S. S. Intille, K. Larson, J. S. Beaudin, J. Nawyn, E. M. Tapia, and P. Kaushik. A living laboratory for the design and evaluation of ubiquitous computing technologies. In CHI '05 Extended Abstracts on Human Factors in Computing Systems, CHI EA '05, pages 1941-1944, New York, NY, USA, 2005. ACM.

[6] D. Kahneman and A. Deaton. High income improves evaluation of life but not emotional well-being. Proc. of the National Academy of Sciences of the United States of America - PNAS, 107(38):16489-16493, 2010.

[7] J. A. Kientz, S. N. Patel, B. Jones, E. Price, E. D. Mynatt, and G. D. Abowd. The georgia tech aware home. In CHI '08 Extended Abstracts on Human Factors in Computing Systems, CHI EA '08, pages 3675-3680, New York, NY, USA, 2008. ACM.

[8] P. Klasnja, S. Consolvo, and W. Pratt. How to evaluate technologies for health behavior change in hci research. In Proceedings of the SIGCHI Conference on Human Factors in Computing Systems, CHI '11, pages 3063-3072, New York, NY, USA, 2011. ACM.

[9] N. D. Lane, M. Lin, M. Mohammod, X. Yang, H. Lu, G. Cardone, S. Ali, A. Doryab, E. Berke, A. T. Campbell, and T. Choudhury. Bewell: Sensing sleep, physical activities and social interactions to promote wellbeing. Mob. Netw. Appl., 19(3):345-359, 2014.

[10] E. Larson, L. Wang, J. Bowen, W. McCormick, L. Teri, P. Crane, and W. Kukull. Exercise is associated with reduced risk for incident dementia among persons 65 years of age and older. Annals of Internal Medicine, 144(2):73-81, 2006.

[11] Y. Lin, J. Jessurun, B. de Vries, and H. Timmermans. Motivate: Context aware mobile application for activity recommendation. In Proc. of the 2nd International Conf. on Ambient Intelligence, pages 210-214, Berlin, Heidelberg, 2011. Springer-Verlag.

[12] M. López-Nores, Y. Blanco-Fernändez, J. J. Pazos-Arias, and J. García-Duque. Exploring synergies between digital tv recommender systems and electronic health records. In Proceedings of EuroITV '10, pages 127-136, New York, NY, USA, 2010. ACM.

[13] F. MF, R. LN, and H. JE. The mini-mental state examination. Archives of General Psychiatry, 40(7):812, 1983.

[14] S. Schnall and J. Laird. Keep smiling: Enduring effects of facial expressions and postures on emotional experience and memory. Cognition and Emotion, 17:787-797, 2003.

[15] E. M. Zelinski, S. E. Dalton, and G. E. Smith. Consumer-based brain fitness programs. In P. E. Hartman-Stein and A. LaRue, editors, Enhancing Cognitive Fitness in Adults, pages 45-66. Springer New York, 2011. 